\title{
ON THE VARIANCE OF LOCAL STEREOLOGICAL VOLUME ESTIMATORS
}

\author{
EVA B VEDEL JENSEN
}

Laboratory for Computational Stchastic Department of Mathematical Sciences University of Aarhus (Accepted November 4, 1999)

\begin{abstract}
In the present paper, the variance of local stereological volume estimators is studied. For isotropic designs, the variance depends on the shape of the body under study and the choice of reference point. It can be expressed in terms of an equivalent star body. For a collection of triaxial ellipsoids the variance is determined by simulation. The problem of estimating particle size distributions from central sections through the particles is also discussed.
\end{abstract}

Keywords: local stereology, particle size distribution, star body, triaxial ellipsoid, variance.

\section{INTRODUCTION}

Local stereology is a collection of sampling designs based on sections through a reference point of the spatial structure under study. Such sections are usually called central sections. The local methods are used in themicroscopical study of biological tissue in cases where the tissue is transparent and physical sections can be replaced by optical sections.

An overview of local stereology has recently been given in Jensen (1998). A comprehensive treatment of local stereological volume estimators can be found in Jensen (1998, p. 105-111). In Jensen and Petersen (1999), it is discussed when the volume of a body in $R^{n}$ can be determined without error by a local stereological estimator and a general variance formula is derived.

In the present paper, we study the variance of local stereological volume estimators for bodies in $R^{3}$. In particular, it is investigated when such estimators can be used in the stereological inference of particle volume distributions.

\section{THE LOCAL VOLUME ESTIMATORS}

Let us start by a short presentation of the local stereological volume estimators. For a body $X \subset R^{n}$, the local stereological estimator of its volume $V(X)$, based on information in an isotropic $p$-subspace $L_{p}$, containing a fixed $r$-subspace $L_{r}$, takes the form

$$
\hat{V}_{n, p, r}\left(X \cap L_{p} ; L_{r}\right)=\pi^{\frac{n-p}{2}} \frac{\Gamma\left(\frac{p-r}{2}\right)}{\Gamma\left(\frac{n-r}{2}\right)} \int_{X \cap L_{p}}\left\|\pi_{L_{r}^{\perp}} x\right\|^{n-p} d x^{p},
$$

where $\|\cdot\|$ is the Euclidean norm, $\pi_{L_{r}^{\perp}}$ is the orthogonal projection onto $L_{r}^{\perp}$ and $d x^{p}$ is the element of $p$-dimensional volume measure in $L_{p}$. When convenient we will use the short notation $\hat{V}_{n, p, r}(X)$ for this estimator.In this paper we will restrict attention to $R^{3}$. In $R^{3}$, there are three local stereological volume estimators which will be considered in more detail below. Without loss of generality, we can assume that the reference point through which the sections pass is the origin $O$.

The first local stereological estimator of $V(X)$ is based on information in an isotropic line $L_{l}$ through $O$ and is given by

$$
\hat{V}_{3,1,0}\left(X \cap L_{1} ; O\right)=2 \pi \int_{X \cap L_{1}}\|x\|^{2} d x^{1} .
$$

If $X \cap L_{1}$ consists of a countable number of linesegments, then

$$
\hat{V}_{3,1,0}\left(X \cap L_{1} ; O\right)=\frac{2}{3} \pi \sum_{x \in \partial X \cap L_{1}}(-1)^{\alpha(x)}\|x\|^{3},
$$

where $\alpha(x)_{x, \partial X \cap L_{1}}$ is a sequence of 0 's and 1's; for details, see Jensen (1998, p. 107-108). In particular, if $X$ is starshaped at $O$ and $\mathrm{O} \in X$, then

$$
\hat{V}_{3,1,0}(X \cap \operatorname{span}\{\omega\} ; O)=\frac{2}{3} \pi\left(\rho_{X}(\omega)^{3}+\rho_{X}(-\omega)^{3}\right)(1)
$$

where $\omega \in S^{2}$, the unit sphere in $R^{3}$, and $\rho_{X}(\omega)$ is the distance from $O$ to the boundary of $X$ in the direction $\omega$ $\in S^{2}$. 
The second local stereological estimator of $V(X)$, based on an isotropic plane $L_{2}$ through $O$, is

$$
\hat{V}_{3,2,0}\left(X \cap L_{2} ; O\right)=2 \int_{X \cap L 2}\|x\| d x^{2} .
$$

This estimator can be obtained as a rotational average of $\hat{V}_{3,1,0}(X)$

$$
\hat{V}_{3,2,0}\left(X \cap L_{2} ; O\right)=E\left(\hat{V}_{3,1,0}\left(X \cap L_{1} ; O\right) \mid L_{2}\right),
$$

where the line $L_{l}$ on the right-hand side of (2) is an isotropic line through $\mathrm{O}$ in $L_{2}$.

The third local volume estimator in $R^{3}$ is based on information in an isotropic plane $L_{2}$, containing a fixed axis $L_{l}$ through $O$. Such a plane is usually called a vertical plane, cf. Baddeley (1984). The estimator takes the form

$$
\hat{V}_{3,2,1}\left(X \cap L_{2} ; L_{1}\right)=\pi \int_{X \cap L_{2}}\left\|\pi_{L_{1}^{L}} x\right\| d x^{2} .
$$

\section{THE ESTIMATOR VARIANCES}

Any of the estimators $\hat{V}_{3,1,0}(X), \hat{V}_{3,2,0}(X)$ and $\hat{V}_{3,2,1}(X)$ are always non-negative and bounded above by $\frac{4 \pi}{3} \rho_{\max }^{3}$ where $\rho_{\max }$ is the maximal distance from $O$ to the boundary of $X$. Accordingly, all three estimators have finite variance. (This appears to be a particularly nice feature of local stereological volume estimators compared to local estimators of lower-dimensional properties such as surface area.) Furthermore, the variance is zero if $X$ is a ball centred at $O$.

Let us study the kind of shapes and choices of origin that imply large respectively small variances of $\hat{V}_{3,1,0}(X)$. To any body $X \subset R^{3}$ we can associate a starshaped (at $O$ ) body $\operatorname{star}(X)$ which is symmetric around $O$ (and therefore contains $O$ ) and has the property that the distribution of its volume estimator $\hat{V}_{3,1,0}(\operatorname{star}(X))$ is the same as for $X$. This body $\operatorname{star}(X)$ will be called the equivalent star body. The body $\operatorname{star}(X)$ is defined by

$\rho_{\text {star }(X)}(\omega)=\left[\frac{3}{4 \pi} \hat{V}_{3,1,0}(X \cap \operatorname{span}\{\omega\} ; O)\right]^{1 / 3},=\omega \in S^{2},(3)$

where $\rho_{\operatorname{star}(X)}(\omega)$ is the distance from $O$ to the boundary of $\operatorname{star}(X)$ in the direction $\omega \in S^{2}$. If we let $B_{r}$ be a ball with centre $O$ and radius $r$, it is easy to see, using (1) with $X$ replaced by $\operatorname{star}(X)$, that

$$
\begin{aligned}
& \hat{V}_{3,1,0}(X \cap \operatorname{span}\{\omega\} ; O)= \\
& \hat{V}_{3,1,0}(\operatorname{star}(X) \cap \operatorname{span}\{\omega\} ; O)= \\
& V\left(B_{\rho_{\text {star }(X)}(\omega)}\right), \omega \in S^{2} .
\end{aligned}
$$

because of the unbiasedness of volume estimators, (4) implies that

$$
V(\operatorname{star}(X))=V(X) .
$$

If $X$ is already star-shaped at $O$ and $O \in X$, the mapping $\mathrm{X} \rightarrow \operatorname{star}(X)$ is a particular type of symmetrization

$$
\rho_{\text {star }(X)}(\omega)=\left[\frac{1}{2} \cdot\left(\rho_{X}(\omega)^{3}+\rho_{X}(-\omega)^{3}\right)\right]^{1 / 3} .
$$

If $X$ is both symmetric and star-shaped at $O$ then $\operatorname{star}(X)=X$. Note that $\operatorname{star}(X)$ does not need to be convex.

Pronounced elongation of $X$ along some lines through $O$ will also be seen in $\operatorname{star}(X)$ and implies large variances. If $O$ is situated asymmetrically in $X$ then $\operatorname{star}(X)$ may show elongation along some lines which again lead to large variances. If $\operatorname{star}(X)$ is a ball then the variance of $\hat{V}_{3,1,0}(X)$ is zero. In Figure 1, an example of a planar section of a body $X$ through $O$ is shown together with the corresponding section of $\operatorname{star}(X)$.

Using (4), it is easy to see that the squared coefficient of error of $\hat{V}_{3,1,0}(X)$ can be expressed in terms of the equivalent star body as

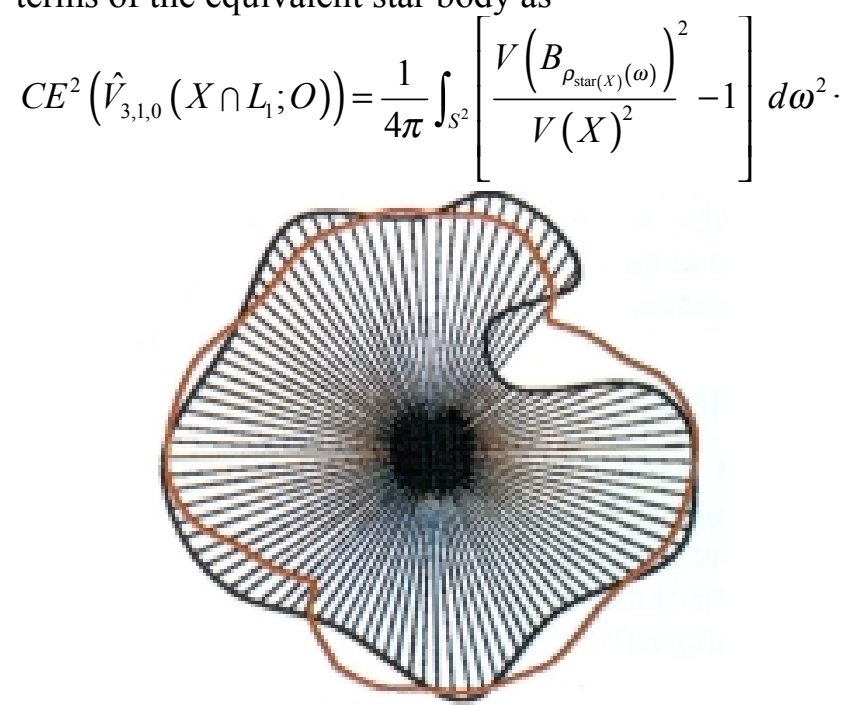

Fig. 1. A planar section of a body $X$ through $O$ (black boundary) together with the corresponding section of star $(X)$ (red boundary). The lines emanating radially from $O$ show that $X$ is not star-shaped at $O$.

Let us now turn to $\hat{V}_{3,2,0}(X)$. Because of (2) 
$\operatorname{Var} \hat{V}_{3,2,0}\left(X \cap L_{2} ; O\right) \leq \operatorname{Var} \hat{V}_{3,1,0}\left(X \cap L_{1} ; O\right)$.

Therefore, if $\operatorname{Var} \hat{V}_{3,1,0}(X)=0$ then $\operatorname{Var} \hat{V}_{3,2,0}(X)=0$. It can also be shown that the opposite statement is correct, cf. Jensen and Petersen (1999, p. 6-8). As for $\hat{V}_{3,1,0}(X)$, it can be shown that the variance of $\hat{V}_{3,2,0}(X)$ depends only on the equivalent star body. We have, cf. Jensen and Petersen (1999, p. 15),

$$
\begin{aligned}
& C E^{2}\left(\hat{V}_{3,2,0}\left(X \cap L_{2} ; O\right)\right)= \\
& \frac{8}{\pi^{3}} \int_{S^{2}} \int_{S^{2}} \sin ^{-1} \angle\left(\omega_{1}, \omega_{2}\right) \\
& \times\left[\frac{V\left(B_{\rho_{\text {star }(X)}\left(\omega_{1}\right)}\right)}{V(X)} \frac{V\left(B_{\rho_{\text {star }(X)}\left(\omega_{2}\right)}\right)}{V(X)}-1\right] d \omega_{2}^{2} d \omega_{1}^{2} .
\end{aligned}
$$

In Table 1, we have determined by simulation the coefficient of error of $\hat{V}_{3,1,0}(X)=0$ and $\hat{V}_{3,2,0}(X)$ for the case where $\operatorname{star}(X)$ is a triaxial ellipsoid centred at $O$ and with semiaxes of lengths $\beta_{1} \geq \beta_{2} \geq \beta_{3}$ and $\beta_{1} / \beta_{2}, \beta_{2} / \beta_{3}$ $\in\{1,2,4\}$. We have also determined the coefficient of error of an intermediate estimator, usually called the nucleator, cf. Gundersen (1988),

$\tilde{V}_{3,1,0}(X)=\frac{1}{2}\left[\hat{V}_{3,1,0}\left(X \cap \operatorname{span}\left\{\omega_{1}\right\} ; O\right)+\hat{V}_{3,1,0}\left(X \cap \operatorname{span}\left\{\omega_{2}\right\} ; O\right)\right]$, where $\omega_{1} \in S^{2} \cap L_{2}$ is an isotropic direction in an isotropic plane $L_{2}$ and $\omega_{2} \in S^{2} \cap L_{2}$ is orthogonal to $\omega_{1}$. Note that a prolate ellipsoid $\left(\beta_{1}=\alpha, \beta_{2}=\beta_{3}=\beta\right)$ gives a larger $C E$ than the corresponding oblate ellipsoid $\left(\beta_{1}=\beta_{2}=\alpha, \beta_{3}=\beta\right)$.

Table 1. Coefficient of error of local stereological volume estimators in various triaxial ellipsoids. For details, see text.

\begin{tabular}{cc|ccc}
$\beta_{1} / \beta_{2}$ & $\beta_{2} / \beta_{3}$ & $\mathrm{CE}\left(\hat{V}_{3,1,0}\right)$ & $\mathrm{CE}\left(\tilde{V}_{3,1,0}\right)$ & $\mathrm{CE}\left(\hat{V}_{3,2,0}\right)$ \\
\hline 1 & 1 & 0 & 0 & 0 \\
1 & 2 & 0.59 & 0.33 & 0.28 \\
1 & 4 & 1.20 & 0.76 & 0.52 \\
\hline 2 & 1 & 0.72 & 0.41 & 0.34 \\
2 & 2 & 1.27 & 0.79 & 0.56 \\
2 & 4 & 2.01 & 1.34 & 0.77 \\
\hline 4 & 1 & 1.85 & 1.21 & 0.77 \\
4 & 2 & 2.74 & 1.86 & 1.02 \\
4 & 4 & 3.98 & 2.76 & 1.25 \\
\hline
\end{tabular}

Similar results can be established for $\hat{V}_{3,2,1}(X)$.

The equivalent star body is here replaced by an equivalent cylinder with axis parallel to the vertical axis and a star-shaped, symmetric base.

In practice, the shape of $X$ is unknown, however. If it is possible, using optical sectioning, for instance, to suggest an extreme shape such that the real distribution of $\hat{V}(X) / V(X)$ is more concentrated around 1 than that obtained under the extreme shape, then conservative $(1-\alpha)$ - confidence limits are

$$
\frac{\hat{V}(X)}{u_{1-\frac{\alpha}{2}}} \leq V(X) \leq \frac{\hat{V}(X)}{u_{\frac{\alpha}{2}}}
$$

where $u_{\alpha}$ is the $100 \alpha$-percentile, determined by simulation, in the distribution of $\hat{V}(X) / V(X)$ under the extreme shape assumption for $X$.

\section{PARTICLE SIZE DISTRIBUTIONS FROM CENTRAL SECTIONS}

Let us consider an aggregate of particles $\left\{X_{i}\right\}$, the object being to estimate the particle size distribution from central sections through the particles. To be more precise, we suppose that a point $x_{i} \in X_{i}$ associated to each $X_{i}$. A central section through $X_{i}$ is then a section containing $x_{i}$. As size parameter, we may take the volume, but other size parameters are possible, see below. For any of the volume estimators, described in the previous section, we have

$$
\frac{\hat{V}(X)}{V\left(X_{i}\right)}=1+\varepsilon_{i},
$$

where the associated point $x_{i} \in X_{i}$ is acting as origin and $\varepsilon_{i}$ has mean zero and a distribution depending on the shape of $X_{i}$ and the relative position of $x_{i}$ in $X_{i}$. In a vertical design, the distribution of $\varepsilon_{i}$ also depends on the choice of vertical axis relative to $X_{i}$. If the confidence limits (5) for each sampled particle are narrow compared to the variability in the volume distribution then the observed distribution of volume estimates can be regarded as a direct observation of the volume distribution. In any case the average of the volume estimates is an unbiased estimate of the mean particle volume.

If the particle aggregate consists of spheroids (either exclusively prolates or oblates) then more information can be obtained from sections through the centres of the spheroids. In contrast to Cruz-Orive $(1976,1978)$ we sample directly from the particle distribution. This fact appears to make the inference more simple and sound.

Consider a spheroid centred at $O$ and with semiaxes of lengths $\alpha$ and $\beta$, where $\alpha \geq \beta$. We will parametrize by (size, shape). For prolates, it is convenient to use 


$$
(y, z)=\left(\beta,\left[1-\frac{\beta^{2}}{\alpha^{2}}\right]^{1 / 2}\right), y>0,0 \leq z<1,
$$

while for oblates

$$
(y, z)=\left(\alpha,\left[\frac{\alpha^{2}}{\beta^{2}}-1\right]^{1 / 2}\right), y>0, z \geq 0 .
$$

A central section through the spheroid is an ellipse, with semiaxes of lengths $\mu$ and $\rho$, say, where $\mu \geq \rho$. Again, we will parametrize by (size, shape), for prolates by

$$
(u, v)=\left(\rho,\left[1-\frac{\rho^{2}}{\mu^{2}}\right]^{1 / 2}\right), u>0,0 \leq v<1,
$$

for oblates by

$$
(u, v)=\left(\mu,\left[\frac{\mu^{2}}{\rho^{2}}-1\right]^{1 / 2}\right), u>0, v \geq 0 .
$$

Then, it can be shown, cf. Cruz-Orive (1976, formulae (2a) and (2b)) that

$$
u=y, v=\sin \theta \cdot z
$$

where $\theta \in\left\lfloor 0, \frac{\pi}{2}\right)$ is the angle that the normal of the section makes with the rotary axis of the spheroid. From (6), it can be seen that the size of the spheroid is observed directly in a central section while the shape is not.

Let us now suppose that the central section is isotropic with a random angle $\Theta$ with density $\sin \theta, \theta \in\left[0, \frac{\pi}{2}\right)$. Using (6), we can express the conditional density of the observed shape in the section $V$ given the size $Y=y$ in terms of the conditional density of the 3D shape $Z$ given the size $Y=y$

$$
f_{V \mid Y}(v \mid y)=\int_{z=v}^{\infty} \frac{v}{z \sqrt{z^{2}-v^{2}}} f_{Z \mid Y}(z \mid y) d z \text {. }
$$

The integral Eq. 7 is of Abel type and can be inverted. Apart from observing the size distribution, we can thus also in principal estimate the conditional distribution of shape given size.

Note also that (6) implies the following moment relationship between (size, shape) of a randomly chosen spheroid, $(Y, Z)$, and that of an isotropic central section through the spheroid, $(U, V)$,

$$
E\left(U^{a} V^{b}\right)=\frac{1}{2} B\left(\frac{b}{2}+1, \frac{1}{2}\right) E\left(Y^{a} Z^{b}\right),
$$

where $B(\cdot, \cdot)$ is the Beta function.

A preliminary report of some of the data (Vedel Jensen, 1999) has been presented at the $\mathrm{X}^{\text {th }}$ International Congress for Stereology, Melbourne, Australia, 1-4 November 1999.

\section{ACKNOWLEDGEMENTS}

I want to thank Asger Hobolth and Michael Kjærgaard Sørensen for skilful technical assistance.

\section{REFERENCES}

Baddeley AJ. Vertical sections (1984). In: Ambartzumian RV, Weil W, eds. Stochastic Geometry, Geometric Statistics, Stereology. Leipzig: Teubner, 43-52.

Cruz-Orive LM (1976). Particle size-shape distributions: the general spheroid problem. I. Mathematical model. J Microsc 107:235-53.

Cruz-Orive LM (1978). Particle size-shape distributions: the general spheroid problem. II. Stochastic model and practical guide. J Microsc 112:153-67.

Gundersen HJG (1988). The nucleator. J Microsc 151:3-21.

Jensen EBV (1998). Local Stereology. London: World Scientific.

Jensen EBV, Petersen L (1999). When are local stereological volume estimators exact? Research report 4, Laboratory for Computational Stochastics, University of Aarhus. 
